\title{
Rituximab-Induced Hypersensitivity Pneumonitis
}

\author{
Adriano R. Tonellia ${ }^{a}$ Richard Lottenberg ${ }^{b}$ Robert W. Allan ${ }^{c}$ P.S. Sriram ${ }^{a}$

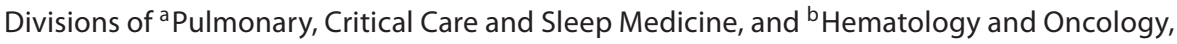 \\ Department of Medicine, and 'Department of Pathology, Immunology and Laboratory Medicine, \\ University of Florida, Gainesville, Fla., USA
}

\section{Established Facts}

- Rituximab has been associated with a variety of respiratory conditions and sporadically with hypersensitivity pneumonitis.

\section{Novel Insights}

- Hypersensitivity pneumonitis is associated with the use of rituximab and it responds to steroids.

\section{Key Words}

Rituximab · Hypersensitivity pneumonitis · Drug-induced lung disease

\begin{abstract}
Rituximab is a chimeric anti-CD20 monoclonal antibody used to treat CD20+ non-Hodgkin's lymphoma. Although pulmonary adverse reactions such as cough, rhinitis, bronchospasm, dyspnea and sinusitis are relatively common, other respiratory conditions like cryptogenic organizing pneumonia, interstitial pneumonitis and diffuse alveolar hemorrhage have rarely been reported. Only 2 possible cases of rituximab-associated hypersensitivity pneumonitis have been described to date. We present a case of hypersensitivity pneumonitis with classic radiographic and histopathologic findings in a patient treated with rituximab who responded to prednisone.

Copyright $\odot 2008$ S. Karger AG, Basel
\end{abstract}

\section{Introduction}

Rituximab is a genetically engineered chimeric (murine/human) anti-CD20 IgG1 monoclonal antibody that is used for the treatment of CD20+ non-Hodgkin's lymphoma.

Side effects of rituximab are commonly seen during the first infusion in up to $50 \%$ of patients and include fever, chills and rigors. These side effects are generally transient and directly related to the tumor burden, probably due to a greater degree of complement activation and proinflammatory cy tokine release [1-6].

Respiratory adverse reactions have been reported in $38 \%$ of patients treated with rituximab in clinical trials, including cough, rhinitis, bronchospasm, dyspnea and sinusitis [4]. A few case reports have described more severe respiratory injuries associated with the use of rituximab, such us cryptogenic organizing pneumonia, interstitial

\section{KARGER \\ Fax +41613061234 E-Mail karger@karger.ch} www.karger.com

\section{(c) 2008 S. Karger AG, Basel}

0025-7931/09/0782-0225\$26.00/0

Accessible online at:

www.karger.com/res
Adriano Tonelli, MD

Health Science Center

1600 SW Archer Road, Room M452

Gainesville, FL 32610-0225 (USA)

Tel. +1 352682 8067, Fax +1 352392 0821, E-Mail Adriano.Tonelli@medicine.ufl.edu 


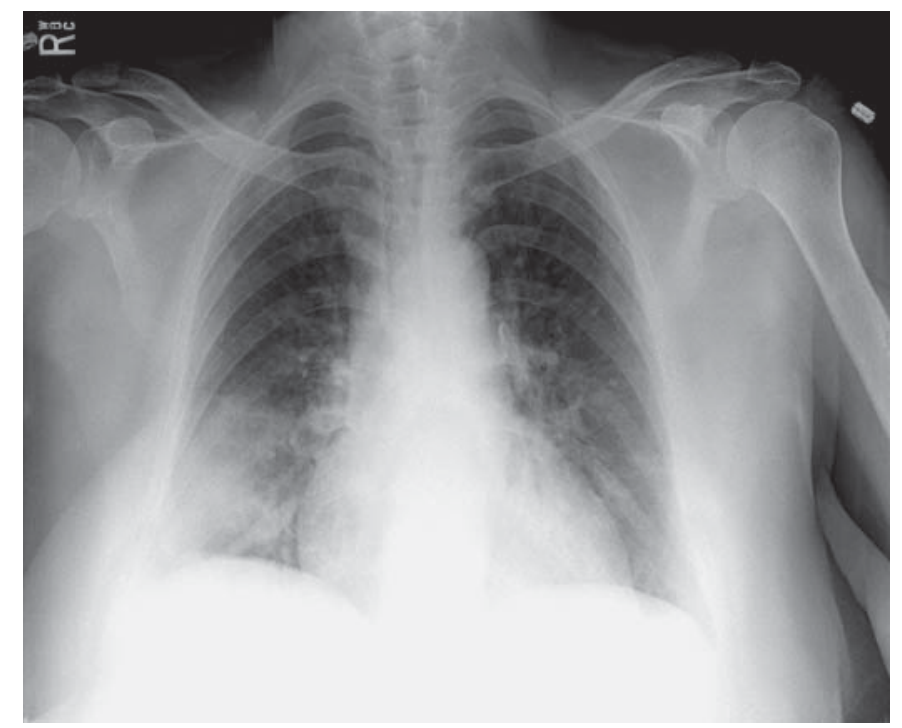

Fig. 1. Chest radiography shows bilateral infiltrates predominantly in the lower lobes.

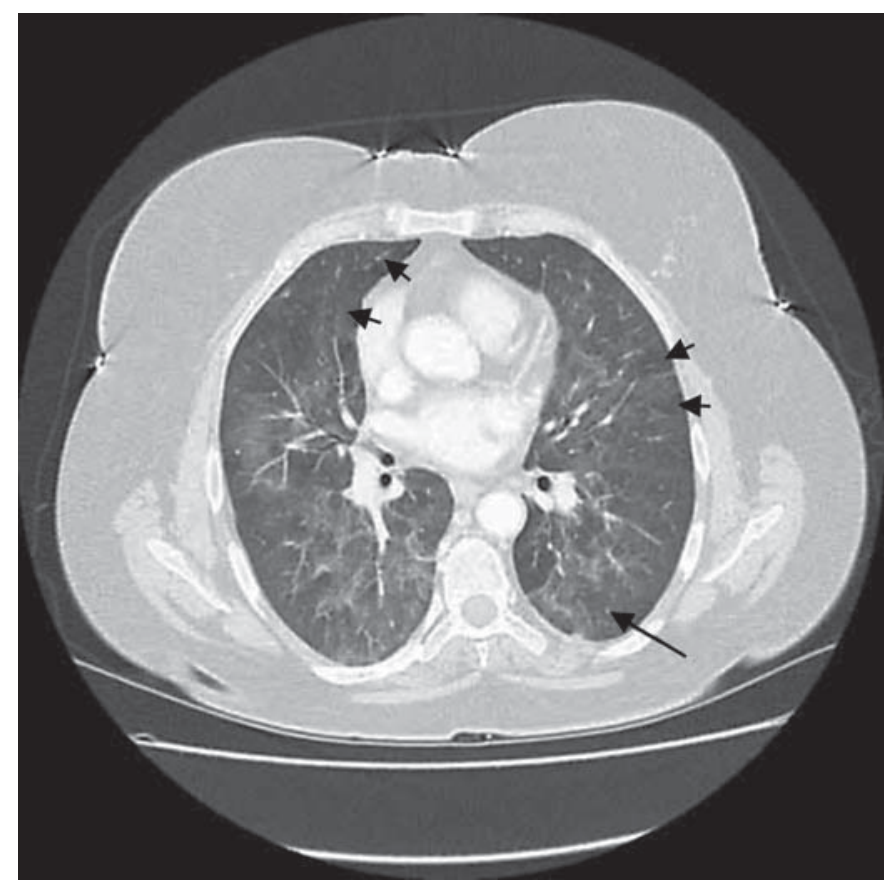

Fig. 2. Chest computed tomography shows ground-glass opacities, small centrilobular nodules (long arrow) and areas of decreased attenuation or mosaic pattern (short arrows). These findings are characteristics radiographic features of subacute hypersensitivity pneumonitis. pneumonitis and diffuse alveolar hemorrhage, some of which resulted in fatal outcomes [4]. According to the manufacturer of the medication, the calculated reporting rate of all possible cases of severe rituximab-induced lung injury is less than $0.03 \%$ [7].

Hypersensitivity pneumonitis is a diffuse parenchymal lung disease characterized by a non-IgE-mediated immunologic reaction to an inhaled allergen. Occasionally, hypersensitivity pneumonitis can be a manifestation of drug-induced lung disease wherein clinical, radiologic and histopathologic features are indistinguishable from those caused by inhaled organic antigens [8].

Rituximab has been sporadically linked to the development of hypersensitivity pneumonitis. Two reports [9, $10]$ in the medical literature have described the presence of histopathological findings suggestive of this condition. We present a case of hypersensitivity pneumonitis associated with rituximab treatment in a patient with chronic lymphocytic leukemia.

\section{Case Report}

A 59-year-old woman presented with symptomatic anemia with a direct antiglobulin test positive for IgG and complement. Evaluation of the warm autoimmune hemolytic anemia included a bone marrow examination that revealed chronic lymphocytic leukemia. Treatment with prednisone at $1 \mathrm{mg} / \mathrm{kg}$ resulted in an inadequate response, and weekly rituximab at $375 \mathrm{mg} / \mathrm{m}^{2}$ was administered 4 times along with continuing steroid therapy. After rituximab was started, the hemoglobin level improved, and over a 6-week period, the prednisone was tapered from 90 to $20 \mathrm{mg}$ daily. Ten days after decreasing the daily prednisone dose to 20 $\mathrm{mg}$, the patient presented with a history of progressive dyspnea and dry cough for about a week. She had received the last dose of rituximab 3.5 weeks before this admission. Her initial vital signs were as follows: temperature $36.9^{\circ} \mathrm{C}$, heart rate 88 beats $/ \mathrm{min}$, blood pressure $140 / 80 \mathrm{~mm} \mathrm{Hg}$, respiratory rate $20 / \mathrm{min}$ and oxygen saturation $91 \%$ on room air. Physical examination was unremarkable except for bilateral scattered inspiratory crackles. Laboratory examination revealed a white blood cell count of 5,900/ $\mathrm{mm}^{3}$ (65.9\% neutrophils, $24.3 \%$ lymphocytes and $1.4 \%$ eosinophils), hemoglobin $11.2 \mathrm{~g} / \mathrm{dl}$, platelet count $243,000 / \mathrm{mm}^{3}$, blood urea nitrogen $40 \mathrm{mg} / \mathrm{dl}$, creatinine $1.2 \mathrm{mg} / \mathrm{dl}$, calcium $10.4 \mathrm{mg} / \mathrm{dl}$ and glucose $92 \mathrm{mg} / \mathrm{dl}$. An HIV ELISA test was negative. Tumor necrosis factor- $\alpha$, determined by multi-analyte fluorescence detection, was undetectable. Chest radiography showed diffuse bilateral lung infiltrates (fig. 1). A computed tomography of the chest revealed diffuse bilateral ground-glass opacities, poorly defined centrilobular nodules and mosaic attenuation (fig. 2). The patient underwent bronchoscopy with bronchoalveolar lavage and transbronchial biopsies. Bronchoalveolar lavage fluid showed 40 red blood cells $/ \mathrm{mm}^{3}$ and 15 white blood cells $/ \mathrm{mm}^{3}$ (80\% monocytes, $15 \%$ lymphocytes and $5 \%$ polymorphonuclear cells). The transbronchial biopsy revealed poorly formed granulomata, with 
an increase in interstitial lymphocytes that were composed of predominantly CD3+/CD5+ lymphocytes, with a predominance of CD4+ over CD8+ T cells as seen by immunohistochemical stains (fig. 3). Special stains for acid-fast bacilli (Kinyoun acid-fast bacilli stain) and fungi/Pneumocystis (Gomori methenamine silver stain) were negative. A cytomegalovirus immunohistochemical stain was negative. Culture was negative for bacteria, fungi and mycobacteria. A meticulous review of the patient's environmental and occupational exposure showed no obvious external cause for hypersensitivity pneumonitis. The patient was treated with prednisone $1 \mathrm{mg} / \mathrm{kg}$ with an excellent clinical response including normalization of the oxygen saturation ( $97 \%$ on room air) within several days. The patient is currently being tapered off prednisone.

\section{Discussion}

Rituximab-induced interstitial lung disease is a rare but known complication. Its low incidence may be attributed to a failure to recognize the complication or resolution either spontaneously after discontinuing the medication or after a course of steroids [11].

In two comprehensive reviews $[10,11]$ of all reported cases of rituximab-induced interstitial lung disease, it is described that most patients were above 55 years old and had either a diagnosis of diffuse large B cell lymphoma or chronic lymphocytic leukemia. The majority of patients presented with progressive dyspnea, cough, fevers and hypoxemia after at least 4 cycles of rituximab. Chest radiographs and computed tomographies often showed diffuse bilateral interstitial infiltrates. Lung biopsies predominantly revealed alveolar damage and interstitial fibrosis. Although spontaneous resolution occurred with discontinuation of rituximab, more than half of the patients required high-dose corticosteroids. The duration of steroid therapy was usually 1-2 months [9-11].

Several confounding factors can affect the interpretation of these results. Firstly, only half of the reports described lung histopathology. Secondly, only a small number of patients were treated with rituximab as a single agent (other chemotherapeutic agents such as cyclophosphamide, doxorubicin, vincristine, bleomycin, videsine, mitoxantrone and etoposide were used in combination). Whether interstitial pneumonitis was a result of rituximab, other chemotherapeutic agents or a combination thereof is difficult to elucidate. Some authors have hypothesized that the pulmonary toxicity of chemotherapeutic agents can be enhanced by concomitant use of rituximab, through a synergistic cytokine activity or by production of deleterious reactive oxygen species $[5,12]$.

Pneumonitis with Rituximab

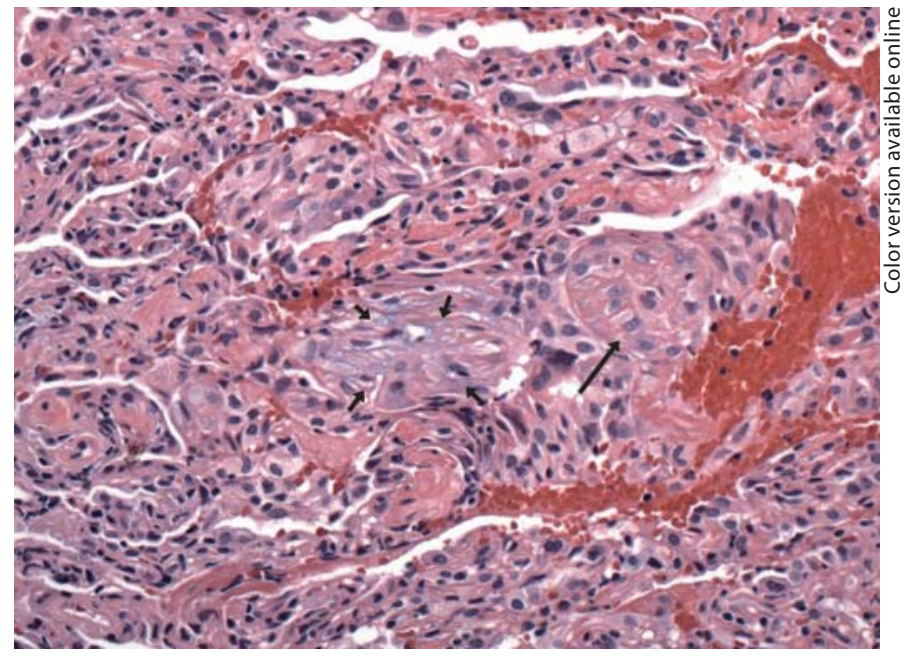

Fig. 3. Transbronchial biopsy $(\mathrm{HE}, \times 400)$ showing an increase in interstitial lymphocytes with a poorly formed granuloma (long arrow) and intraluminal fibrous plug (short arrows).

Hypersensitivity pneumonitis represents an immunologic reaction that has not been explicitly associated with rituximab treatment; however, 2 case reports have described findings suggestive of this condition, as they point out the presence of loose non-necrotizing granulomas in a background of lymphocytic infiltrate $[9,10]$.

The first report [9] described a 65-year-old man with diffuse B cell lymphoma who received 5 cycles of rituximab and cyclophosphamide, doxorubicin, vincristine and prednisone (CHOP). He presented with cough, dyspnea, macular rash, fever, hypoxemia and eosinophilia. The patient initially responded to prednisone $40 \mathrm{mg} / \mathrm{day}$, but deteriorated after the 6th cycle of CHOP without rituximab. The ground-glass opacities progressed and the patient required mechanical ventilation and finally died of sepsis and multiorgan failure. Autopsy revealed intra-alveolar hemorrhage with diffuse alveolar damage along with loosely formed granulomas in a background of lymphocytic infiltrate.

The second report [10] described an 88-year-old man with Waldenstrom's macroglobulinemia who had received fludarabine and cyclophosphamide more than 3 years prior to presentation and had recently been given rituximab (8 doses). Eight weeks after the last dose of rituximab he experienced progressive dyspnea, cough and hemoptysis associated with hypoxemia, eosinophilia and bilateral alveolar/interstitial infiltrates on a chest computed tomography. Bronchoalveolar lavage fluid was suggestive of diffuse alveolar hemorrhage and was lym-

Respiration 2009;78:225-229 
phocyte predominant. Transbronchial biopsy showed interstitial pneumonitis with scattered, loosely formed granulomas suggestive of a hypersensitivity-like reaction. The patient improved dramatically within 4 days of starting prednisone $60 \mathrm{mg} /$ day.

Although both patients had histopathology suggestive of hypersensitivity pneumonitis, they also had peripheral eosinophilia and elevated IgE, which are not usually seen in true hypersensitivity pneumonitis.

Our patient had distinctive clinical and radiological findings in the absence of external causes of hypersensitivity pneumonitis. Our patient did not have peripheral eosinophilia in the blood and she was not receiving treatment with other chemotherapeutic agents that may have obscured the presentation.

Although the bronchoalveolar lavage in hypersensitivity pneumonitis is often lymphocyte predominant with a decrease in the CD4+/CD8+ ratio, in our case the bronchoalveolar lavage showed mononuclear cell predominance, and biopsy showed an increase in the CD4+/ $\mathrm{CD} 8+$ ratio. Bronchoalveolar lavage findings may support the likelihood of certain lung diseases; however, the information is not specific, and in order to obtain a definitive diagnosis, further investigations are required [13, 14]. In drug-associated pneumonitis, a predominantly lymphocytic fluid is the most common finding in the bronchoalveolar lavage, but there are cases with normal cell differential [15]. Although the bronchoalveolar lavage fluid in our patient showed mononuclear cell predominance, the transbronchial biopsies evidenced a predominance of lymphocytes. While the lymphocyte $\mathrm{CD} 4+/ \mathrm{CD} 8+$ ratio is usually reduced in hypersensitivity pneumonitis, a broad range of possible CD4+/CD8+ lymphocyte ratios has been reported $[14,16]$.

Of the 2 previous reports describing possible rituximab-associated hypersensitivity pneumonitis $[9,10]$, one patient evidenced an increased $\mathrm{CD} 4+/ \mathrm{CD} 8+$ ratio in the lung tissue [9] as in our case and the other showed a predominantly lymphocytic bronchoalveolar lavage fluid (CD4+/CD8+ ratio was not reported) [10].

The transbronchial biopsy in our patient was compatible with subacute hypersensitivity pneumonitis as it had all the necessary histological features for the diagnosis, i.e. bronchiolocentric interstitial pneumonitis with interstitial lymphocytic infiltrates, cellular bronchiolitis and poorly formed (loose) non-necrotizing granulomas [17].

It is unclear why our patient developed hypersensitivity pneumonitis while on low-dose prednisone. It is possible that low-dose prednisone $(20 \mathrm{mg} /$ day) was inadequate to suppress the development of pneumonitis. This theory might be supported by a case report of a patient on rituximab for refractory immune thrombocytopenic purpura who developed interstitial pneumonitis while on high-dose prednisolone therapy (60 mg/day) [18].

\section{Conclusion}

In patients receiving rituximab, hypersensitivity pneumonitis, though rare, should be considered in the appropriate clinical and radiographic setting. Rituximab should be discontinued; complete and rapid resolution is possible with systemic steroids.

\section{References}

1 Bienvenu J, Chvetzoff R, Salles G, Balter C, Tilly H, Herbrecht R, Morel P, Lederlin P, Solal-Celigny P, Audhuy B, Christian B, Gabarre J, Casasnovas O, Marit G, Sebban C, Coiffier B; Groupe d'Etude des Lymphomes de l'Adulte: Tumor necrosis factor alpha release is a major biological event associated with rituximab treatment. Hematol J 2001;2: 378-384.

2 Winkler U, Jensen M, Manzke O, Schulz H, Diehl V, Engert A: Cytokine-release syndrome in patients with B-cell chronic lymphocytic leukemia and high lymphocyte counts after treatment with an anti-CD20 monoclonal antibody (rituximab, IDECC2B8). Blood 1999;94:2217-2224.

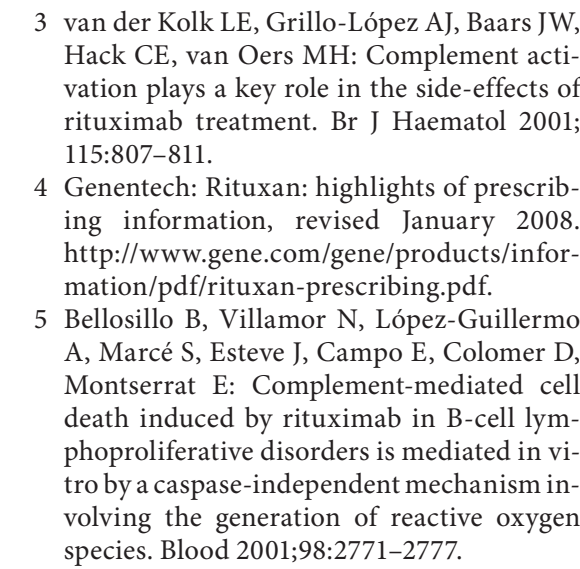

6 Wu SJ, Chou WC, Ko BS, Tien HF: Severe pulmonary complications after initial treatment with rituximab for the Asian-variant of intravascular lymphoma. Haematologica 2007;92:141-142.

7 Burton C, Kaczmarski R, Jan-Mohamed R: Interstitial pneumonitis related to rituximab therapy. N Engl J Med 2003;348:2690-2691.

$\checkmark 8$ Patel AM, Ryu JH, Reed CE: Hypersensitivity pneumonitis: current concepts and future questions. J Allergy Clin Immunol 2001;108: 661-670.

$\checkmark 9$ Alexandrescu DT, Dutcher JP, O’Boyle K, Albulak M, Oiseth S, Wiernik PH: Fatal intra-alveolar hemorrhage after rituximab in a patient with non-Hodgkin lymphoma. Leuk Lymphoma 2004;45:2321-2325. 
$>_{10}$ Heresi GA, Farver CF, Stoller JK: Interstitial pneumonitis and alveolar hemorrhage complicating use of rituximab. Case report and review of the literature. Respiration 2008; 76:449-453.

11 Wagner SA, Mehta AC, Laber DA: Rituximab-induced interstitial lung disease. Am J Hematol 2007;82:916-919.

$\checkmark 12$ Ghesquieres H: Severe interstitial pneumonitis following rituximab and bleomycincontaining combination chemotherapy. Ann Oncol 2005;16:1399.
3 Costabel U, Uzaslan E, Guzman J: Bronchoalveolar lavage in drug-induced lung disease. Clin Chest Med 2004;25:25-35.

14 Welker L, Jörres RA, Costabel U, Magnussen $\mathrm{H}$ : Predictive value of BAL cell differentials in the diagnosis of interstitial lung diseases. Eur Respir J 2004;24:1000-1006.

$>15$ Akoun GM, Cadranel JL, Rosenow EC 3rd, Milleron BJ: Bronchoalveolar lavage cell data in drug-induced pneumonitis. Allerg Immunol (Paris) 1991;23:245-252.
16 Girard M, Israël-Assayag E, Cormier Y: Pathogenesis of hypersensitivity pneumonitis. Curr Opin Allergy Clin Immunol 2004; 4:93-98.

17 Silva CI, Churg A, Müller NL: Hypersensitivity pneumonitis: spectrum of high-resolution CT and pathologic findings. AJR Am J Roentgenol 2007;188:334-344.

18 Swords R, Power D, Fay M, O’Donnell R, Murphy PT: Interstitial pneumonitis following rituximab therapy for immune thrombocytopenic purpura (ITP). Am J Hematol 2004;77:103-104. 